\title{
Neutrino photoproduction on the electron in dense magnetized medium
}

\author{
Alexander Kuznetsov ${ }^{1, \star}$, Dmitriy Rumyantsev ${ }^{1, \star \star}$, and Denis Shlenev ${ }^{1, \star \star \star}$ \\ ${ }^{1}$ Yaroslavl State University
}

\begin{abstract}
The process of neutrino photoproduction on an electron, $e \gamma \rightarrow e v \bar{v}$, in a strongly magnetized cold plasma in resonant case has been considered. The contribution of this process to the neutrino emissivity has been calculated. It has been shown that under such conditions neutrino emissivity due to process $e \gamma \rightarrow e v \bar{v}$ could be expressed in terms of emissivity of it's subprocess, $e \rightarrow e v \bar{v}$.
\end{abstract}

\section{Introduction}

Nowadays, there exists rather keen interest to astrophysical objects with the scale of the magnetic field strength near the critical value of $B_{e}=m^{2} / e \simeq 4.41 \times 10^{13} \mathrm{G}$ (we use natural units $c=\hbar=k_{\mathrm{B}}=1, m$ is the electron mass, and $e$ is the elementary charge). This group of objects includes the radio pulsars and the so-called magnetars, which are the neutron stars featuring the magnetic field strengths from $10^{12} \mathrm{G}$ (radio pulsars) to $4 \times 10^{14} \mathrm{G}$ (magnetars), see Ref. [1] and the papers cited therein. The spectra analysis of these objects also provides an evidence for the presence of electron-positron plasma in the radio pulsar and magnetar environment, with the minimum magnetospheric plasma density being of the order of the Goldreich-Julian density [2]:

$$
n_{G J} \simeq 3 \times 10^{13} \mathrm{~cm}^{-3}\left(\frac{B}{100 B_{e}}\right)\left(\frac{10 \mathrm{~s}}{P}\right),
$$

where $P$ is the rotational period. It is well-known that strong magnetic field and/or plasma could have an essential influence on various quantum processes [3-6], because the external active medium catalyses the processes, by changing their kinematics and inducing new interactions. Therefore, the effects of magnetized plasma on microscopic physics should be incorporated in the magnetosphere models of strongly magnetized neutron stars.

All known theoretical models of internal structure of neutron stars give the parameters of the medium (density and temperature) at which the magnetized plasma is transparent to neutrinos. In this case, reactions with a neutrino-antineutrino pair in the final state are decisive for neutrino cooling. In this connection, the neutrino photoproduction (the so called photoneutrino process), $e \gamma \rightarrow e v \bar{v}$, was studied by multiple authors. In papers [7-9] the formulas for neutrino emissivity, i.e. energy carried away by the neutrino pair from the unit volume of a star by unit time, were calculated for

\footnotetext{
^e-mail: avkuzn@uniyar.ac.ru

$\star \star$ e-mail: rda@uniyar.ac.ru

$\star \star \star$ e-mail: allen_caleb@rambler.ru
} 
both nonrelativistic and relativistic plasmas. However, in those papers the anisotropy in the dispertion of photons was disregarded, which can change the results essentially. In recent paper [10] neutrino emisivity was calculated with taking into account the dispertion properties of photon in plasma in non-resonant case.

Into the boundary between inner and outer crust of magnetar, where plasma density may exceed value $\rho_{9}=10^{9} \mathrm{~g} / \mathrm{sm}^{3}$, the higher Landau levels of virtual electron are begin to be excited and the resonance on virtual electron may occur.

In this work we study in detail the process of photoproduction of neutrino-antineutrino pair, $e \gamma \rightarrow$ $e v \bar{v}$, and the neutrino emissivity owing to this process in dense magnetized medium in resonance case.

\section{Neutrino emissivity}

A general expression for neutrino emissivity can be defined as follows:

$$
Q=\frac{1}{V} \int \prod_{i} \mathrm{~d} \Gamma_{i} f_{i} \prod_{f} \mathrm{~d} \Gamma_{f}\left(1 \pm f_{f}\right) q_{0}^{\prime} \frac{\left|S_{i f}\right|^{2}}{\tau},
$$

where $\mathrm{d} \Gamma_{i}\left(\mathrm{~d} \Gamma_{f}\right)$ are the number of states of initial (final) particles; $f_{i}\left(f_{f}\right)$ are the corresponding distribution functions, the sign $+(-)$ corresponds to final bosons (fermions); $q_{0}^{\prime}$ is the neutrino pair energy; $V$ is the plasma volume, $\tau$ is the interaction time, $S_{\text {if }}$ is the $S$-matrix element.

To calculate $S$-matrix element of photoneutrino process we will utilize Lagrangian for electroweak interaction in the case of relatively small momentum transfers compared with $W$ boson mass, $\left|q^{\prime 2}\right| \ll$ $m_{W}^{2}$, where $q^{\prime}$ is the four-vector of neutrino pair. Under this condition, the effective Lagrangian can be written as follows:

$$
\mathcal{L}=\frac{G_{F}}{\sqrt{2}}\left[\bar{\Psi} \gamma_{\alpha}\left(C_{V}-C_{A} \gamma_{5}\right) \Psi\right] j_{\alpha}+e\left(\bar{\Psi} \gamma_{\alpha} \Psi\right) A_{\alpha}
$$

where $j_{\alpha}=\bar{v} \gamma_{\alpha}\left(1-\gamma_{5}\right) v$ is the neutrino current, $A_{\alpha}$ is the four-potential of the photon field, $C_{V}=$ $\pm 1 / 2+2 \sin ^{2} \theta_{W}, C_{A}= \pm 1 / 2$, and $\theta_{W}$ is the Weinberg angle. Here, the upper sign corresponds to electron neutrinos $\left(v=v_{e}\right)$, when there is exchange reaction both of $W$ and $Z$ bozons. The lower sign corresponds to the $\mu$ and $\tau$ neutrinos, when there is only $Z$ bozon exchange.

The $S$-matrix element in the tree-level approximation is described by Feynman diagrams shown in Fig. 1. It should be noted that the resonance on the virtual electron occurs in the $s$-channel (the first diagram in Fig.1) only. In this case we can present the $S$-matrix element in the next form:

$$
\mathcal{S}_{e \gamma \rightarrow e v \bar{v}}^{s^{\prime} s}=\frac{e G_{f}}{\sqrt{2}} \int \mathrm{d}^{4} X \mathrm{~d}^{4} Y j_{\alpha}^{*}(Y) A_{\beta}(X)\left[\bar{\Psi}_{p^{\prime} l^{\prime}}^{s^{\prime}}(Y) \gamma_{\alpha}\left(C_{V}-C_{A} \gamma_{5}\right) \hat{S}(Y, X) \gamma_{\beta} \Psi_{p l}^{s}(X)\right]
$$

The currents in Eq. (4) can be expressed through the amplitudes in the momentum space:

$$
\begin{aligned}
& A_{\beta}(X)=\frac{e^{-\mathrm{i}(q X)}}{\sqrt{2 q_{0} V}} A_{\beta}(q), \\
& j_{\alpha}(Y)=\frac{e^{-\mathrm{i}\left(q^{\prime} Y\right)}}{\sqrt{2 q_{0}^{\prime} V}} j_{\alpha}\left(q^{\prime}\right) .
\end{aligned}
$$




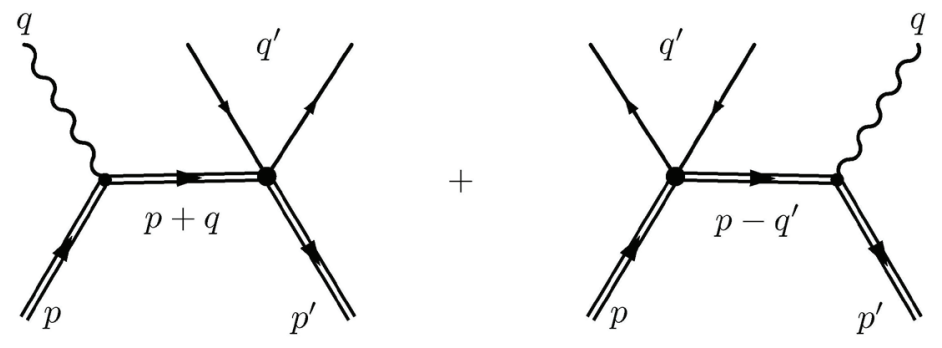

Figure 1. The Feynman diagrams for the reaction $\gamma e \rightarrow e v \bar{v}$. Double lines mean that the effects of external field on the initial and the final electron states and on the electron propagator are exactly taken into account.

There exist several descriptions of the procedure of obtaining the electron wave functions in the presence of an external magnetic field by solving the Dirac equation, see e.g. Refs. [14]-[20] and also Refs. [5,6]. In the most cases, the solutions are presented in the form with the upper two components of the bispinor corresponding to the electron states with the spin projections $1 / 2$ and $-1 / 2$ on the magnetic field direction. Here, we have found it more convenient to use another representation of the electron wave functions, being the eigenstates of the magnetic moment operator $\mu_{z}[16,17]$

$$
\mu_{z}=m \Sigma_{z}-\mathrm{i} \gamma_{0} \gamma_{5}[\boldsymbol{\Sigma} \times \boldsymbol{P}]_{z},
$$

where $\boldsymbol{P}=-\mathrm{i} \boldsymbol{\nabla}+\boldsymbol{e A}$. We take the frame where the field is directed along the $z$ axis, and the Landau gauge where the four-potential is: $A^{\lambda}=(0,0, x B, 0)$. It is convenient to use the notation $\beta=e B$, and to introduce the sign of the fermion charge, $\eta$.

Our choice of the Dirac equation solutions as the eigenfunctions of the operator $\hat{\mu}_{z}$ is caused by the following arguments. Calculations of the process widths with two or more vertices in an external magnetic field by the standard method, including the squaring the amplitude with all the Feynman diagrams and with summation or averaging over the electron polarization states, contain significant computational difficulties. In this case, it is convenient to calculate partial contributions to the amplitude from the channels with different electron polarization states and for each diagram separately, by direct multiplication of the bispinors and the Dirac matrices. The result, up to a total for both diagrams non-invariant phase, will have an explicit Lorentz invariant structure. On the contrary, the amplitudes obtained with using the solutions for a fixed direction of the spin, do not have Lorentz invariant structure. Only the amplitude squared, summed over the electron polarization states, is manifestly Lorentz-invariant with respect to a boost along the magnetic field direction.

The electron wave functions having the form

$$
\Psi_{p, n}^{s}(X)=\frac{e^{-\mathrm{i}\left(E_{n} X_{0}-p_{y} X_{2}-p_{z} X_{3}\right)} U_{n}^{s}(\xi)}{\sqrt{4 E_{n} M_{n}\left(E_{n}+M_{n}\right)\left(M_{n}+m\right) L_{y} L_{z}}},
$$

where

$$
E_{n}=\sqrt{M_{n}^{2}+p_{z}^{2}}, \quad M_{n}=\sqrt{m^{2}+2 \beta n},
$$

are the solutions of the equation

$$
\hat{\mu}_{z} \Psi_{p, n}^{s}(X)=s M_{n} \Psi_{p, n}^{s}(X), \quad s= \pm 1 .
$$


It is convenient to present the bispinors $U_{n}^{s}(\xi)$ in the form of decomposition over the solutions for negative and positive fermion charge, $U_{n, \eta}^{s}(\xi)$ :

$$
U_{n}^{s}(\xi)=\frac{1-\eta}{2} U_{n,-}^{s}(\xi)+\frac{1+\eta}{2} U_{n,+}^{s}(\xi),
$$

where

$$
\begin{aligned}
& U_{n,-}^{-}(\xi)=\left(\begin{array}{c}
-\mathrm{i} \sqrt{2 \beta n} p_{z} V_{n-1}(\xi) \\
\left(E_{n}+M_{n}\right)\left(M_{n}+m\right) V_{n}(\xi) \\
-\mathrm{i} \sqrt{2 \beta n}\left(E_{n}+M_{n}\right) V_{n-1}(\xi) \\
-p_{z}\left(M_{n}+m\right) V_{n}(\xi)
\end{array}\right), \\
& U_{n,-}^{+}(\xi)=\left(\begin{array}{c}
\left(E_{n}+M_{n}\right)\left(M_{n}+m\right) V_{n-1}(\xi) \\
-\mathrm{i} \sqrt{2 \beta n} p_{z} V_{n}(\xi) \\
p_{z}\left(M_{n}+m\right) V_{n-1}(\xi) \\
\mathrm{i} \sqrt{2 \beta n}\left(E_{n}+M_{n}\right) V_{n}(\xi)
\end{array}\right), \\
& U_{n,+}^{-}(\xi)=\left(\begin{array}{c}
\mathrm{i} \sqrt{2 \beta n} p_{z} V_{n}(\xi) \\
\left(E_{n}+M_{n}\right)\left(M_{n}+m\right) V_{n-1}(\xi) \\
\mathrm{i} \sqrt{2 \beta n}\left(E_{n}+M_{n}\right) V_{n}(\xi) \\
-p_{z}\left(M_{n}+m\right) V_{n-1}(\xi)
\end{array}\right) \\
& U_{n,+}^{+}(\xi)=\left(\begin{array}{c}
\left(E_{n}+M_{n}\right)\left(M_{n}+m\right) V_{n}(\xi) \\
\mathrm{i} \sqrt{2 \beta n} p_{z} V_{n-1}(\xi) \\
p_{z}\left(M_{n}+m\right) V_{n}(\xi) \\
-\mathrm{i} \sqrt{2 \beta n}\left(E_{n}+M_{n}\right) V_{n-1}(\xi)
\end{array}\right),
\end{aligned}
$$

$V_{n}(\xi)(n=0,1,2, \ldots)$ are the normalized harmonic oscillator functions, which are expressed in terms of the Hermite polynomials $H_{n}(\xi)[21]$ :

$$
\begin{aligned}
& V_{n}(\xi)=\frac{\beta^{1 / 4} \mathrm{e}^{-\xi^{2} / 2}}{\sqrt{2^{n} n ! \sqrt{\pi}}} H_{n}(\xi), \\
& \xi=\sqrt{\beta}\left(X_{1}-\eta \frac{p_{y}}{\beta}\right) .
\end{aligned}
$$

Let us define functions $\phi_{p \ell}^{s}\left(X_{1}\right)$ (index $p$ corresponds to electron momentum), based on bispinors $U_{\ell}^{s}(\xi)$ :

$$
\phi_{p \ell}^{s}\left(X_{1}\right)=\frac{U_{\ell}^{s}\left[\xi\left(X_{1}\right)\right]}{\sqrt{2 M_{\ell}\left(E_{\ell}+M_{\ell}\right)\left(M_{\ell}+m\right)}} .
$$


We use the electron propagator in the form of the sum over the Landau levels [6, 22]:

$$
\begin{gathered}
\hat{S}\left(X, X^{\prime}\right)=\sum_{n=0}^{\infty} \hat{S}_{n}\left(X, X^{\prime}\right), \\
\hat{S}_{n}\left(X, X^{\prime}\right)=\sum_{s= \pm 1} \hat{S}_{n}^{s}\left(X, X^{\prime}\right), \\
\hat{S}_{n}^{s}\left(X, X^{\prime}\right)=\int \frac{\mathrm{d} p_{0} \mathrm{~d} p_{y} \mathrm{~d} p_{z}}{(2 \pi)^{3}} \frac{\mathrm{e}^{-\mathrm{i}\left(p\left(X-X^{\prime}\right)\right)_{\|}+\mathrm{i} p_{y}\left(X_{2}-X_{2}^{\prime}\right)}}{p_{\|}^{2}-M_{n}^{2}-\mathcal{R}_{\Sigma}^{s}(p)+\mathrm{i} \mathcal{I}_{\Sigma}^{s}(p)} \phi_{p n}^{s}\left(X_{1}\right) \bar{\phi}_{p n}^{s}\left(X_{1}^{\prime}\right),
\end{gathered}
$$

where $\xi$ is defined by Eq. (17) and $\xi^{\prime}$ is obtained from $\xi$ by substituting $X_{1} \rightarrow X_{1}^{\prime}$.

Hereafter we use the following notations: four-vectors with the indices $\perp$ and $\|$ belong to the Euclidean $\{1,2\}$ subspace and the Minkowski $\{0,3\}$ subspace correspondingly. Then for arbitrary 4-vectors $A_{\mu}, B_{\mu}$ one has

$$
\begin{aligned}
& A_{\perp}^{\mu}=\left(0, A_{1}, A_{2}, 0\right), \quad A_{\|}^{\mu}=\left(A_{0}, 0,0, A_{3}\right), \\
& (A B)_{\perp}=(A \Lambda B)=A_{1} B_{1}+A_{2} B_{2}, \\
& (A B)_{\|}=(A \widetilde{\Lambda} B)=A_{0} B_{0}-A_{3} B_{3},
\end{aligned}
$$

where the matrices $\Lambda_{\mu v}=(\varphi \varphi)_{\mu v}, \widetilde{\Lambda}_{\mu v}=(\tilde{\varphi} \tilde{\varphi})_{\mu v}$ are constructed with the dimensionless tensor of the external magnetic field, $\varphi_{\mu \nu}=F_{\mu \nu} / B$, and the dual tensor, $\tilde{\varphi}_{\mu v}=\frac{1}{2} \varepsilon_{\mu \nu \rho \sigma} \varphi^{\rho \sigma}$. The matrices $\Lambda_{\mu \nu}$ and $\widetilde{\Lambda}_{\mu v}$ are connected by the relation $\widetilde{\Lambda}^{\mu \nu}-\Lambda^{\mu \nu}=g^{\mu \nu}=\operatorname{diag}(1,-1,-1,-1)$, and play the roles of the metric tensors in the perpendicular $(\perp)$ and the parallel $(\|)$ subspaces respectively.

Real part $\mathcal{R}_{\Sigma}^{s^{\prime \prime}}(P)$ and imaginary part $\mathcal{I}_{\Sigma}^{s^{\prime \prime}}(P)$ of eigenvalue of electron mass operator are the result of calculation of radiative corrections to the electron mass, caused by combined action of magnetic field and plasma. The imaginary part $\mathcal{I}_{\Sigma}^{s^{\prime \prime}}(P)$ can be obtained from the results of $[11,12]$ and has the following form:

$$
\mathcal{I}_{\Sigma}^{s^{\prime \prime}}(P)=-\frac{1}{2} P_{0} \Gamma_{n}^{s^{\prime \prime}}
$$

where $\Gamma_{n}^{s^{\prime \prime}}$ is the total width of the change of electron state. This width can be expressed in terms of the width of electron creation [23]:

$$
\begin{aligned}
& \Gamma_{n}^{s^{\prime \prime}}=\Gamma_{n}^{(a b s) s^{\prime \prime}}+\Gamma_{n}^{(c r) s^{\prime \prime}} \simeq \Gamma_{e_{n} \rightarrow e_{\ell^{\prime}} \gamma}^{(c r) s^{\prime \prime}}\left[1+\mathrm{e}^{\left(E_{n}^{\prime \prime}-\mu\right) / T}\right] \\
& \Gamma_{n}^{(c r) s^{\prime \prime}}=\sum_{\ell=0}^{n-1} \sum_{s^{\prime \prime}} \frac{1}{2 E_{n}^{\prime \prime}} \int \frac{d^{3} k}{2 q_{0}(2 \pi)^{3}} f_{\gamma}\left(q_{0}\right) \frac{d^{2} p}{2 E_{\ell}} f_{e}\left(E_{\ell}\right)(2 \pi)^{3} \delta^{(3)}\left(P-p^{\prime \prime}\right)\left|M_{e_{\ell} \gamma \rightarrow e_{n}}\right|^{2}
\end{aligned}
$$

where $f_{\gamma}\left(q_{0}\right)$ is the equillibrium distribution fuction of initial photon, $f_{e}\left(E_{\ell}\right)$ is the equillibrium distribution fuction of initial electron in the plasma rest frame.

Real part of mass operator, $\mathcal{R}_{\Sigma}^{s^{\prime \prime}}(P)$, determines the change of the electron dispertion properties in presence of magnetized plazma. It was shown in $[5,18]$ that mass corrections are negligibly small under conditions $B \lesssim 10^{16} \mathrm{G}$. 
After integration in Eq. (4) over $\mathrm{d}^{4} X$ and $\mathrm{d}^{4} Y$ we obtain:

$$
S_{e \gamma \rightarrow e v \bar{\nu}}^{s^{\prime} s}=\frac{\mathrm{i}(2 \pi)^{3} \delta^{(3)}\left(P-p^{\prime}-q^{\prime}\right)}{\sqrt{2 q_{0} V 2 q_{0}^{\prime} V 2 E_{\ell} L_{y} L_{z} 2 E_{\ell^{\prime}}^{\prime} L_{y} L_{z}}} \mathcal{M}_{e \gamma \rightarrow e v \bar{\nu}}^{s^{\prime} s},
$$

where $\delta^{3}\left(P-p^{\prime}-q^{\prime}\right)=\delta\left(P_{0}-E_{\ell^{\prime}}^{\prime}-q_{0}^{\prime}\right) \delta\left(P_{y}-p_{y}^{\prime}-q_{y}^{\prime}\right) \delta\left(P_{z}-p_{z}^{\prime}-q_{z}^{\prime}\right), P_{\alpha}=(p+q)_{\alpha}, \alpha=0,2,3$, and the amplitude $\mathcal{M}_{e \gamma \rightarrow e v \bar{v}}^{s^{\prime} s}$ can be presented in the following form:

$$
\begin{aligned}
& \mathcal{M}_{e \gamma \rightarrow e v \bar{v}}^{s^{\prime} s}=\mathrm{i} \frac{e G_{F}}{\sqrt{2}} \sum_{n=0}^{\infty} \sum_{s^{\prime \prime}} j_{\alpha}^{*}\left(q^{\prime}\right) A_{\beta}(q) \times \\
& \times \int \mathrm{d} X_{1} \mathrm{~d} Y_{1} \mathrm{e}^{-\mathrm{i} X_{1} q_{x}+\mathrm{i} Y_{1} q_{x}^{\prime}} \frac{\bar{\phi}_{p^{\prime} \ell^{\prime}}^{s^{\prime}}\left(Y_{1}\right) \gamma_{\alpha}\left(C_{V}-C_{A} \gamma_{5}\right) \phi_{P n}^{s^{\prime \prime}}\left(Y_{1}\right) \phi_{P n}^{s^{\prime \prime}}\left(X_{1}\right) \gamma_{\beta} \phi_{p \ell}^{s}\left(X_{1}\right)}{P_{\|}^{2}-M_{n}^{2}+\mathrm{i} \mathcal{I}_{\Sigma}^{s^{\prime \prime}}(P)\left(X_{1}\right)}
\end{aligned}
$$

Assuming that the main contribution to amplitude is given by resonance region, we can approximately replace the part of the amplitude by the $\delta$-function [13]:

$$
\begin{aligned}
& \left|\mathcal{M}_{e \gamma \rightarrow e v \bar{\gamma}}^{s^{\prime} s}\right|^{2} \simeq \frac{\left(e G_{F}\right)^{2}}{2} \sum_{n=0}^{\infty} \sum_{s^{\prime \prime}} \frac{\pi}{P_{0} \Gamma_{n}^{s^{\prime \prime}}} \delta\left(P_{\|}^{2}-M_{n}^{2}\right) \mid j_{\alpha}\left(q^{\prime}\right) A_{\beta}(q) \times \\
& \times\left.\int \mathrm{d} X_{1} \mathrm{~d} Y_{1} \bar{\phi}_{p^{\prime} \ell^{\prime}}^{s^{\prime}}\left(Y_{1}\right) \gamma_{\alpha}\left(C_{V}-C_{A} \gamma_{5}\right) \phi_{P n}^{s^{\prime \prime}}\left(Y_{1}\right) \phi_{P n}^{s^{\prime \prime}}\left(X_{1}\right) \gamma_{\beta} \phi_{p \ell}^{s}\left(X_{1}\right)\right|^{2} .
\end{aligned}
$$

After applying the delta-finction properties:

$$
\delta\left(P_{\|}^{2}-M_{n}^{2}\right)=\frac{1}{2 E_{n}^{\prime \prime}} \delta\left(P_{0}-E_{n}^{\prime \prime}\right)
$$

where $E_{n}^{\prime \prime}=\sqrt{p_{z}^{\prime \prime 2}+M_{n}^{2}}$, it is straightforward to show that amplitude squared, averaged over polarizations of initial photon can be factored by amplitudes of sub-processes:

$$
\left|\mathcal{M}_{\gamma e \rightarrow e v \bar{\nu}}\right|^{2} \simeq \sum_{n=1}^{\infty} \frac{\pi}{P_{0} \Gamma_{n}^{s^{\prime \prime}}} \delta\left(P_{\|}^{2}-M_{n}^{2}\right)\left|\mathcal{M}_{e_{\ell} \gamma \rightarrow e_{n}}\right|^{2}\left|\mathcal{M}_{e_{n} \rightarrow e^{\prime}} \nu \bar{v}\right|^{2}
$$

Substituing Eq. (29)into the expression for the the luminocity (2), we obtain:

$$
Q_{\gamma e \rightarrow e v \bar{\nu}}=\sum_{n=1}^{\infty} \sum_{\ell^{\prime}=0}^{n-1} Q_{e_{n} \rightarrow e^{\prime}},
$$

where

$$
\begin{aligned}
& Q_{e_{n} \rightarrow e_{\ell^{\prime}} \nu \bar{v}}=\frac{1}{L_{x}} \int \frac{\mathrm{d} p_{y}^{\prime \prime} \mathrm{d} p_{z}^{\prime \prime}}{(2 \pi)^{2} 2 E_{n}^{\prime \prime}} f_{e}\left(E_{n}^{\prime \prime}\right) \frac{\mathrm{d} p_{y}^{\prime} \mathrm{d} p_{z}^{\prime}}{(2 \pi)^{2} 2 E_{\ell^{\prime}}^{\prime}}\left[1-f_{e}\left(E_{\ell^{\prime}}^{\prime}\right)\right] \times \\
& \times \frac{\mathrm{d}^{3} p_{1}}{(2 \pi)^{3} 2 E_{1}} \frac{\mathrm{d}^{3} p_{2}}{(2 \pi)^{3} 2 E_{2}} q_{0}^{\prime}(2 \pi)^{3} \delta_{0, y, z}^{3}\left(p^{\prime \prime}-p^{\prime}-q^{\prime}\right)\left|\mathcal{M}_{e_{n} \rightarrow e_{\ell^{\prime}} \nu \bar{v}}\right|^{2}
\end{aligned}
$$


is the neutrino luminocity due to process $e_{n} \rightarrow e_{\ell^{\prime}} v \bar{v}$ [24]. Squared amplitude of the process $e_{n} \rightarrow e_{\ell^{\prime}} v \bar{v}$ has the form:

$$
\begin{aligned}
& \left.\left|\mathcal{M}_{e_{n} \rightarrow \ell_{\ell^{\prime}}}\right|^{2}\right|^{2}=G_{\mathrm{F}}^{2}\left(\overline{C_{V}^{2}}+\overline{C_{A}^{2}}\right)\left\{\left[2 q^{2}\left(\beta(n+\ell)+m^{2}\right)+m^{2} q_{\perp}^{2}\right] \times\right. \\
& \times\left(\mathcal{I}_{n-1, \ell}^{2}+\mathcal{I}_{n, \ell-1}^{2}-\mathcal{I}_{n, \ell}^{2}-\mathcal{I}_{n-1, \ell-1}^{2}\right)-q^{4}\left(\mathcal{I}_{n-1, \ell}^{2}+\mathcal{I}_{n, \ell-1}^{2}\right)+ \\
& \left.+m^{2} q^{2}\left(\mathcal{I}_{n, \ell}^{2}-\mathcal{I}_{n-1, \ell-1}^{2}\right)\right\}-G_{\mathrm{F}}^{2}\left(\overline{C_{V}^{2}}-\overline{C_{A}^{2}}\right) m^{2}\left\{\left(2 q_{\|}^{2}-q_{\perp}^{2}\right) \times\right. \\
& \left.\times\left(\mathcal{I}_{n-1, \ell}^{2}+\mathcal{I}_{n, \ell-1}^{2}-\mathcal{I}_{n, \ell}^{2}-\mathcal{I}_{n-1, \ell-1}^{2}\right)+3 q^{2}\left(\mathcal{I}_{n-1, \ell}^{2}+\mathcal{I}_{n, \ell-1}^{2}\right)\right\} .
\end{aligned}
$$

Here $I_{n, \ell} \equiv I_{n, \ell}\left(q_{\perp}^{2} /(2 \beta)\right), I_{n, \ell}(x)=\sqrt{\frac{\ell !}{n !}} \mathrm{e}^{-x / 2} x^{(n-\ell) / 2} L_{\ell}^{n-\ell}(x)$. and $L_{n}^{k}(x)-$ generalized Laguerre polinomials [21], The constants $\overline{C_{V}^{2}}=0.93$ and $\overline{C_{A}^{2}}=0.75$ are the results of summation over all channels of the neutrino production of the types $v_{e}, v_{\mu}, v_{\tau}$.

The obtained luminocity (31) coincides up to notation with results of [24]. It also generalizes obtained in [13] expression to the case of arbitrary initial and final electron Landau level.

\section{Conclusion}

We have considered the neutrino photoproduction on an electron, $e \gamma \rightarrow e v \bar{v}$, in dense magnetized medium in resonant case.

It has been shown that in the case of resonance on the virtual electron, the neutrino emissivity due to the process $\gamma e_{\ell} \rightarrow e_{\ell^{\prime}} v \bar{v}$ can be expressed in terms of the neutrino emissivity due to the process $e_{n} \rightarrow e_{\ell^{\prime}} v \bar{v}$. Obtained results coincide up to notation with obtained earlier in [24]. They also generalize the results of recent studies [13] to the case, when initial and final electron occupy arbitrary Landau level.

\section{References}

[1] S. A. Olausen \& V. M. Kaspi, Astrophys. J. Suppl. 212, 6 (2014).

[2] P. Goldreich \& W. H. Julian, Astrophys. J. 157, 869 (1969).

[3] D. Lai, Rev. Mod. Phys. 73, 629 (2001).

[4] A. K. Harding \& D. Lai, Rept. Prog. Phys. 69, 2631 (2006).

[5] A. V. Kuznetsov \& N. V. Mikheev, Electroweak Processes in External Electromagnetic Fields (Springer-Verlag, New York, 2003).

[6] A. V. Kuznetsov \& N. V. Mikheev, Electroweak Processes in External Active Media (SpringerVerlag, Berlin, Heidelberg, 2013).

[7] D.A. Rumyantsev and M.V. Chistyakov J. Exp. Theor. Phys. 107, 533 (2008)

[8] A.V. Borisov, B.K. Kerimov and P. E. Sizin, in: Particle Physics at the Tercentenary of Mikhail Lomonosov, Proc. 15th Lomonosov Conf. on Elementary Particle Physics, Moscow, 2011. Ed. A.I. Studenikin. World Sci., Singapore, 2013, p. 204-205

[9] A.V. Borisov, B.K. Kerimov and P. E. Sizin Phys. At.Nucl. 75 (11), 1305 (2012)

[10] N.V. Mikheev, D.A. Rumyantsev, M.V. Chistyakov J. Exp. Theor. Phys. 146, 289 (2014) 
[11] A. V. Borisov, A. S. Vshivtsev, V. C. Zhukovsky, and P. A. Eminov, Phys. Usp. 40, 229 (1997)

A. V. Kuznetsov, D. A. Rumyantsev, and D. M. Shlenev, Int. J. Mod. Phys. A 30, 1550049 (2015)

[12] V. C. Zhukovsky, P. G. Midodashvili, and P. A. Eminov, JETP 79, 509 (1994)

[13] A. V. Kuznetsov, D. A. Rumyantsev, and D. M. Shlenev, Int. J. Mod. Phys. A 30, 1550049 (2015)

[14] M. H. Johnson \& B. A. Lippmann, Phys. Rev. 76, 828 (1949).

[15] A. I. Akhiezer \& V. B. Berestetskii, Quantum Electrodynamics. 2nd ed. (Wiley, New York 1965).

[16] A. A. Sokolov \& I. M. Ternov, Synchrotron Radiation (Pergamon, Oxford, 1968).

[17] D. B. Melrose \& A. J. Parle, Aust. J. Phys. 36, 755 (1983).

[18] A. A. Sokolov \& I. M. Ternov, Radiation from Relativistic Electrons (American Institute of Physics, New York 1986).

[19] K. Bhattacharya \& P. B. Pal, Pramana J. Phys. 62, 1041 (2004).

[20] I. A. Balantsev, Yu. V. Popov, \& A. I. Studenikin, J. Phys. A 44, 255301 (2011).

[21] I. S. Gradshteyn \& I. M. Ryzhik, Table of Integrals, Series, and Products (Academic, New York, 1980).

[22] A. V. Kuznetsov \& A. A. Okrugin, Int. J. Mod. Phys. A 26, 2725 (2011).

[23] H. A. Weldon, Phys. Rev. D 28, 2007 (1983).

[24] D.G. Yakovlev, A.D. Kaminker, O.Y. Gnedin and P. Haensel, Phys. Rep. 354, 1 